\title{
Opioid-induced hyperalgesia after implantation of intrathecal morphine pump: a case report
}

\author{
Sungryul Yoon ${ }^{1}$, Rakmin Choi ${ }^{2}$, Jaedo Lee ${ }^{1}$, Jongchan Won', Bousung Lee ${ }^{1}$, Kwanghaeng Lee ${ }^{1}$, \\ Dongseok Kim ${ }^{1}$, Jaeyoung Yang ${ }^{1}$
}

Department of Anesthesiology and Pain Medicine, 'Veterans Health Service Medical Center, Seoul, ${ }^{2}$ Armed Forces Capital Hospital, Seongnam, Korea

\author{
Received March 6, 2020 \\ Revised April 10, 2020 \\ Accepted May 28, 2020 \\ Corresponding author \\ Jaeyoung Yang \\ Department of Anesthesiology \\ and Pain Medicine, Veterans \\ Health Service Medical Center, 53 \\ Jinhwangdo-ro 61-gil, Gangdong- \\ gu, Seoul 05368, Korea \\ Tel: +82-2-2225-1473 \\ Fax: +82-2-2225-1946 \\ E-mail: yang303@gmail.com \\ ORCID: \\ https://orcid.org/0000-0002-9923-6712
}

\begin{abstract}
Opioid-induced hyperalgesia is characterized by an increased pain response to noxious stimuli despite increased use of opioid medications. Here, we report the case of a 43-year-old woman diagnosed with post-laminectomy syndrome who presented with an increasing pain score following a morphine infusion via an implanted intrathecal drug delivery device. Her pain improved after reducing opioid doses and the administration of intravenous ketamine infusion therapy. Thus, the early suspicion of opioid-induced hyperalgesia is essential for patients with increasing pain refractory to augmented doses of opioid medications.
\end{abstract}

Keywords: Hyperalgesia; Implantable; Intrathecal; Ketamine; Morphine; Opioid

\section{INTRODUCTION}

Post-laminectomy syndrome, a relatively common condition caused by back surgery, can decrease patient quality of life. The prevalence of post-laminectomy syndrome is reportedly up to $60 \%$ [1]. Because post-laminectomy syndrome can be difficult to treat, the use of a multimodal approach consisting of psychological support, physical therapy, anti-inflammatory medication, oral opioid, and nerve block is necessary. Advanced pain management devices such as spinal cord stimulators and intrathecal (IT) morphine pumps can be used to treat intractable post-laminectomy syndrome $[2,3]$. Consensus is lacking regarding post-laminectomy syn- drome management. Narcotic analgesics are often effective for post-laminectomy syndrome patients, although controversy persists regarding their long-term use. Physicians can become embarrassed if a patient's pain score worsens while on opioid management. When opioid-related complications are suspected, various causes such as opioid withdrawal, toxicity, tolerance, and hyperalgesia should be investigated. Distinguishing opioid tolerance from the deterioration of a pre-existing condition is another daunting challenge.

Opioid-induced hyperalgesia (OIH), an uncommon phenomenon observed in patients receiving opioid therapy, occurs after patients use various opioid medications such as remifentanil, fentanyl, sufentanil, and oxycodone [4-7]. Di- 
verse routes of administration such as oral, intravenous (IV), epidural, and IT are associated with OIH. However, the diagnosis of $\mathrm{OIH}$ is often delayed or misdiagnosed because of its low incidence and vague appearance as well as delayed clinical recognition.

Here we present a case of a 43-year-old female who developed $\mathrm{OIH}$ after receiving a morphine infusion through an implanted IT drug delivery device. Here, we share our clinical experience successfully managing a case of $\mathrm{OIH}$.

\section{CASE REPORT}

The patient, a 43-year-old woman with diabetes mellitus, underwent a lumbosacral fusion operation 6 years prior to treat a herniated disc. At 2 months postoperative, she developed lower back pain radiating to the bilateral lower extremities with a visual analog scale (VAS) score of 8 . She was diagnosed with post-laminectomy syndrome and underwent spinal cord stimulator implantation 3 months later. She subsequently underwent a series of neuraxial and sympathetic ganglion blocks that did not effectively resolve her pain. Signs of depression manifested. She recently developed a sleep disturbance because of the increasing pain. Her opioid medication dose was gradually increased to $440 \mathrm{mg}$ of oral oxycodone daily and $100 \mu \mathrm{g}$ of transdermal fentanyl hourly equivalent to $840 \mathrm{mg}$ of daily oral morphine $(8.4 \mathrm{mg}$ of IT morphine daily). Her non-opioid medications included gabapentin, baclofen, afloqualone, duloxetine, quetiapine, clonazepam, and mirtazapine. However, these drugs were unable to mitigate her pain. The patient agreed to undergo the implantation of an IT drug delivery device. The patient provided written informed consent for the publication of this case report, which was approved by the Institutional Review Board of the Veterans Health Service Medical Center (2020-01-014).

After she was admitted to our hospital, she received an effective IT bolus of $0.3 \mathrm{mg}$ of morphine that reduced her VAS pain score to 4 . Although it is general practice to taper at least $50 \%$ of the dose of previous opioid medications before a trial [2,8], we maintained her original medications because she strongly refused to reduce them due to her extreme fear of enduring more pain than she was already experiencing. IT drug delivery device implantation was scheduled. Under general anesthesia, the catheter was inserted through L4-L5.
The catheter tip was located at the level of the T11 vertebral body. The pump was placed in a subcutaneous pocket in the right abdominal region and connected to the catheter via an extension cable through a subcutaneous tunnel. The initial infusion rate was set to $1.4 \mathrm{mg}$ of morphine daily. Her overall VAS pain score decreased to 4 on the same day.

Three days later, the patient reported pain worsening. The IT infusion rate was gradually increased three times throughout the following 8 days until it reached $2.0 \mathrm{mg}$ of daily morphine. Nevertheless, the pain severity increased to a VAS score of 9. The patient complained of widespread pain involving her trunks and upper extremities. Several diagnostic measures including laboratory findings, X-rays of relevant regions, and a neurologic examination were performed. However, these tests failed to identify any causes of the increased pain score. A contrast study of the implanted device confirmed that it was functionally intact without catheter obstruction. The likelihood of $\mathrm{OIH}$ was considered. After the pain physician's decision was explained, her oral and transdermal opioid medication doses were reduced by $50 \%$ and her VAS pain score decreased to 4 .

The following day, she complained of severe pain with a VAS score of 10. Thus, an IV ketamine infusion was administered. Treatment with $2 \mathrm{mg}$ of midazolam followed by $20 \mathrm{mg}$ of ketamine and $60 \mathrm{mg}$ of ketamine infused over 1 hour immediately decreased her VAS pain score to 6 . Three consecutive identical ketamine infusions administered over the next 10 days of the hospitalization ameliorated her VAS pain score to a mean of 4 . The patient was discharged on postoperative day 25 . Additionally, $200 \mathrm{mg}$ of oxycodone and $50 \mu \mathrm{g}$ of transdermal fentanyl hourly were prescribed for the patient. She was satisfied with the new quality of life provided by the IT morphine pump device.

\section{DISCUSSION}

Post-laminectomy syndrome is a common challenging condition encountered by physicians managing spinal disorders owing to the expanded indications for spinal surgery. Various factors can lead to post-laminectomy syndrome: preoperative factors include smoking, obesity, and depression, while postoperative factors include new-onset foraminal stenosis, recurrent disc herniation, muscular atrophy, muscle spasm, and degenerative changes to the postopera- 
tive spine [0].

A multidisciplinary approach is recommended for post-laminectomy syndrome patients. For pharmacological therapy, paracetamol and nonsteroidal anti-inflammatory drugs are recommended for axial back pain [10]. Anticonvulsant drugs such as gabapentin and pregabalin can effectively alleviate neuropathic pain. Gabapentin is superior to naproxen at managing back pain [11]. The use of opioids in post-laminectomy syndrome is quite controversial; in fact, it is currently recommended only for short-term therapy [12]. In neuromodulation therapy, the spinal cord stimulator is the most effective treatment for neuropathic limb pain. $\mathrm{Ku}^{-}$ mar reported that $48 \%$ of the spinal cord stimulator group versus $9 \%$ of the conventional medical management group achieved at least a 50\% reduction in pain score [3].

The IT drug delivery system was first released for cancer chemotherapy in 1988 by Medtronic. The indications for the device were expanded to cancer- and non-cancer-related pain in 1991 [13]. The system consists of an implantable drug storage device and an IT catheter. The drug delivery mode can be programed by an external device positioned over the implanted pump. The American Society of Interventional Pain Physicians recommends reserving the IT drug delivery system as the last management tool. The IT drug delivery system can reportedly be used for various chronic pain conditions such as post-laminectomy syndrome, compression fracture, spinal stenosis, complex regional pain syndrome, rheumatoid arthritis, connective tissue disorders, and chronic pancreatitis [13]. The device's analgesic response outcomes were reportedly quite favorable (up to 73\%) [14].

Various IT drug delivery systems feature complications such as bleeding, infection, catheter malfunction, neurologic injury, cerebral spinal fluid leakage, inflammatory mass, and central hypocortisolism [15]. In the present case, the patient's neuropathic pain could not be controlled with regular pain medications. A spinal cord stimulator was also unable to control her symptoms. High-dose opioids was the only modality that provided short-term pain relief.

Paradoxical hyperalgesia was observed in the absence of a careful dosing strategy in the present case. The general protocol is to taper $50-100 \%$ of the previous opioid dosage for several weeks prior to the trial and completely wean opioid medications at least 1 week before implantation $[2,8]$.
However, our patient refused a reduction in the dose of oral opioid medication for IT morphine pump implantation. The increase in opioid dosage using the IT morphine pump might have led to the paradoxical $\mathrm{OIH}$.

In IT morphine pump-implanted patients, clinical conditions related to IT opioid infusion are complicated and difficult to treat. When exacerbation of neuropathic pain is observed in a patient taking high-dose opioids, it is necessary to distinguish among various etiologies such as opioid withdrawal, toxicity, tolerance, and hyperalgesia. Opioid withdrawal resulting from the sudden cessation of opioid infusion can be confirmed with pump testing using contrast dye. It is usually accompanied by other symptoms such as nausea, vomiting, or an altered sense of smell [16]. The presence of myoclonus and rigor after each bolus shot from the IT morphine pump could suggest opioid central toxicity [16]. Exposure to opioids can lead to increased doses over time to maintain an analgesic effect, known as tolerance. Tolerance reflects the desensitization of antinociceptive pathways to opioids. However, OIH reflects a sensitization of the pronociceptive pathways [17]. OIH can be observed in IT morphine pump-implanted patients [16]. Opioid tolerance-related pain could be reduced by increasing the opioid dosage. OIH can be confirmed when exacerbated pain can be alleviated by a dosage reduction or opioid antagonist application. However, distinguishing between opioid tolerance and $\mathrm{OIH}$ in clinical practice can be challenging.

In the present case, it was unclear if the cause of the $\mathrm{OIH}$ was the oral or IT opioid. It might be reasonable to deduce that additional IT morphine might enhance the sensitization of the pronociceptive pathways to opioids. The mechanisms responsible for the development of $\mathrm{OIH}$ are complex and not completely understood. N-methyl-D-aspartate receptors, $\mu$-receptors, and genetic factors are proposed mechanisms [18-20].

The clinical suspicion of $\mathrm{OIH}$ is the most crucial part of the diagnosis. In $\mathrm{OIH}$, as a response to escalating opioid doses, the pain score does not improve; rather, it is exacerbated. However, there is no confirmative test for the diagnosis of $\mathrm{OIH}$. Elon suggested clinical criteria for diagnosing $\mathrm{OIH}$ [21]. He claimed that the following six criteria must be fulfilled for the diagnosis of OIH: increased pain intensity during opioid medication, no evidence of other disease progression, no evidence of opioid withdrawal, no evidence 
of opioid tolerance, no evidence of drug addiction, and a decrease in pain intensity in response to an opioid dose reduction.

Our patient met the above criteria. The exacerbation of her pain was relieved after the opioid dose reduction. Opioid withdrawal, tolerance, and progression of other diseases are other possible causes. She had no opioid withdrawal symptoms such as anxiety, excessive sweating, or lacrimation.

Using multimodal analgesia with non-opioid analgesics combined with an appropriate nerve block and psychosocial techniques can reduce the need for opioids and lower the risk of $\mathrm{OIH}$ [18]. Opioid rotation (conversion to other opioids) could also facilitate the management of $\mathrm{OIH}$. A change of IT morphine to sufentanil or ziconotide was reportedly effective at relieving $\mathrm{OIH}[16,22]$.

Once the diagnosis of $\mathrm{OIH}$ is strongly suspected, patient education and a cautious dose reduction of opioid medications are initial measures. Ketamine infusion therapy is an additional option for $\mathrm{OIH}[18,19]$.

In conclusion, a careful oral opioid reduction is essential in cases of IT morphine pump implantation in which sudden exacerbation of the pain score is observed in patients taking high doses of opioid. OIH should be suspected; once diagnosed, it can be managed with opioid reduction, opioid conversion, and ketamine infusion therapy.

\section{CONFLICT OF INTEREST}

No potential conflict of interest relevant to this article was reported.

\section{REFERENCES}

1. Garcia JB, Rodrigues DP, Leite DR, do Nascimento Câmara S, da Silva Martins K, de Moraes ÉB. Clinical evaluation of the post-laminectomy syndrome in public hospitals in the city of São Luís, Brazil. BMC Res Notes 2015;8:451.

2. Hamza M, Doleys D, Wells M, Weisbein J, Hoff J, Martin M, et al. Prospective study of 3-year follow-up of low-dose intrathecal opioids in the management of chronic nonmalignant pain. Pain Med 2012;13:1304-13.

3. Kumar K, Taylor RS, Jacques L, Eldabe S, Meglio M, Molet J, et al. Spinal cord stimulation versus conventional medical management for neuropathic pain: a multicentre randomised controlled trial in patients with failed back surgery syndrome. Pain 2007;132:179-88.

4. Kim SH, Stoicea N, Soghomonyan S, Bergese SD. Remifentanil-acute opioid tolerance and opioid-induced hyperalgesia: a systematic review. Am J Ther 2015;22:e62-74.

5. Kalaydjian A, Farah F, Cheng Y, Acquadro MA, Gerges FJ. Opioid induced hyperalgesia with intrathecal infusion of high-dose fentanyl. Pain Pract 2019;19:222-3.

6. Devulder J. Hyperalgesia induced by high-dose intrathecal sufentanil in neuropathic pain. J Neurosurg Anesthesiol 1997;9:146-8.

7. Kaneria A. Opioid-induced hyperalgesia: when pain killers make pain worse. BMJ Case Rep 2014;2014:bcr2014204551.

8. Grider JS, Harned ME, Etscheidt MA. Patient selection and outcomes using a low-dose intrathecal opioid trialing method for chronic nonmalignant pain. Pain Physician 2011;14:343-51.

9. Baber Z, Erdek MA. Failed back surgery syndrome: current perspectives. J Pain Res 2016;9:979-87.

10. Chou R, Huffman LH; American Pain Society; American College of Physicians. Medications for acute and chronic low back pain: a review of the evidence for an American Pain Society/American College of Physicians clinical practice guideline. Ann Intern Med 2007;147:505-14.

11. Khosravi MB, Azemati S, Sahmeddini MA. Gabapentin versus naproxen in the management of failed back surgery syndrome; a randomized controlled trial. Acta Anaesthesiol Belg 2014;65:31-7.

12. Franklin GM; American Academy of Neurology. Opioids for chronic noncancer pain: a position paper of the American Academy of Neurology. Neurology 2014;83:1277-84.

13. Wilkes D. Programmable intrathecal pumps for the management of chronic pain: recommendations for improved efficiency. J Pain Res 2014;7:571-7.

14. Angel IF, Gould HJ Jr, Carey ME. Intrathecal morphine pump as a treatment option in chronic pain of nonmalignant origin. Surg Neurol 1998;49:92-8; discussion 98-9.

15. Knight KH, Brand FM, Mchaourab AS, Veneziano G. Implantable intrathecal pumps for chronic pain: highlights and updates. Croat Med J 2007;48:22-34.

16. Singla A, Stojanovic MP, Chen L, Mao J. A differential diagnosis of hyperalgesia, toxicity, and withdrawal from intrathecal morphine infusion. Anesth Analg 2007;105:1816-9, table of contents.

17. Angst MS, Clark JD. Opioid-induced hyperalgesia: a qualitative systematic review. Anesthesiology 2006;104:570-87.

18. Colvin LA, Bull F, Hales TG. Perioperative opioid analgesia-when is enough too much? A review of opioid-induced tolerance and hyperalgesia. Lancet 2019;393:1558-68.

19. Yi P, Pryzbylkowski P. Opioid induced hyperalgesia. Pain 
Med 2015;16 Suppl 1:S32-6.

20. Lee M, Silverman SM, Hansen H, Patel VB, Manchikanti L. A comprehensive review of opioid-induced hyperalgesia. Pain Physician 2011;14:145-61.

21. Eisenberg E, Suzan E, Pud D. Opioid-induced hyperalgesia $(\mathrm{OIH})$ : a real clinical problem or just an experimental phe- nomenon? J Pain Symptom Manage 2015;49:632-6.

22. Webster LR. The relationship between the mechanisms of action and safety profiles of intrathecal morphine and ziconotide: a review of the literature. Pain Med 2015;16:126577 . 